\title{
Active vibration isolation using an induced strain actuator with application to automotive seat suspensions
}

\author{
Mark Malowicki and Donald J. Leo* \\ Center for Intelligent Material Systems and \\ Structures, Mechanical Engineering Department, \\ Virginia Polytechnic Institute and State University, \\ Blacksburg, VA, USA
}

Active vibration isolation of automotive seats requires actuators that achieve millimeter-range displacements and forces on the order of $300 \mathrm{~N}$. Recent developments in piezoceramic actuator technology provide a means for achieving these force and displacement levels in a compact device. This work demonstrates that prestressed, curved piezoceramic actuators achieve the force and displacement levels required for active isolation of automotive seats. An estimate of the force and displacement requirements are obtained from numerical simulations on a four-degree-of-freedom car and seat model that utilize representive road accelerations as inputs. An actuator that meets these specifications is designed using piezoceramic materials. Free displacement of $4.4 \mathrm{~mm}$ and blocked force greater than $300 \mathrm{~N}$ are measured. The actuator is integrated within a dead mass setup that simulates the isolation characteristics of an automotive seat. Control experiments demonstrate that active vibration is achievable with realistic road disturbances. Feedback control is able to eliminate any amplification due to mechanical resonance and reduce the isolation frequency from $9.5 \mathrm{~Hz}$ to $2 \mathrm{~Hz}$.

\section{Introduction}

Vibration isolation in existing seat designs is achieved passively through foam padding placed beneath the seat frame and the passenger. Recent tests on a passenger seat of a luxury sedan demonstrated that the foam padding was the primary mechanism for reducing the transmission of floor vibrations to the pas-

${ }^{*}$ Corresponding author: 307 NEB, Mechanical Engineering Department, Virginia Tech, Blacksburg, VA, 24061-0261, USA. Tel.: +1 540231 2917; Fax: +1 540231 2903; E-mail: donleo@vt.edu. senger in both the translational and rotational degrees of freedom $[12,13]$.

This paper investigates an alternative approach to providing vibration isolation in automotive seats. We investigate the use of a piezoelectric actuator to provide active vibration isolation. It has been shown through analysis and experiment that active isolation overcomes many of the inherent limitations of passive isolation systems. Beard et al. [3] demonstrated that absolute velocity feedback overcomes the constraint between damped response and high-frequency vibration isolation. More recently, Leo and Inman [11] demonstrated that active-passive isolation can achieve significant improvements over passive control when the input energy is concentrated near the break frequency of the isolation system.

The advantages of active control approaches have lead to the experimental demonstration of the technology in several industries. Miller et al. [16] demonstrated the development of active-passive vibration isolation system consisting of a fluid mount and an electromagnetic actuator sized for applications in jet aircraft. More recently, magneto-rheological fluids have been utilized to develop 'semi-active' mounts for vibration isolation [1]. Active-passive vibration isolation has been experimentally demonstrated using voice coil actuators for precision pointing and steering suppression [19]. Anderson et al. [2] discuss the development of a hardmount vibration isolation system using electromagnetic actuators for precision instruments on spacecraft.

Applications of piezoceramic actuator technology have been limited to vibration isolation systems that required sub-millimeter strokes. The primary area of application has been micron-level vibration isolation systems for space environments. Bohannan et al. [5] have demonstrated the use of piezopolymer films for spaceborne vibration isolation systems.

This work contributes to the field of active isolation by demonstrating the feasibility of using piezo- 
electric actuators as control elements in automotive applications. In contrast to space applications, vibration isolation in automotive systems requires displacements on the order of $1-10 \mathrm{~mm}$. Piezoelectric actuators are typically not considered for applications which require millimeter-range displacements due to their low achievable strain $(\approx 0.1 \%)$, but recent work in the field of actuator development has overcome many of these limitations. Several actuator designs have been developed that achieve displacements on the order of 1$5 \mathrm{~mm}$, including mechanically-amplified devices and prestressed curved actuators. Near [18] presents a clear summary of the various actuator types and compares the tradeoffs between force and stroke for piezoelectric devices. Other types of actuators have been developed since Near's overview, including recurve-type [6] and telescoping designs [4], and frequency-rectified devices using piezohydraulic elements $[14,15,17]$.

The fundamental tradeoff in piezoelectric actuator design is between actuator stroke and actuator force. Increasing the displacement of piezoelectric actuators is accompanied by a reduction in force. Assessing the feasibility of a piezoelectric actuator requires a model of the system and an estimate of the force and displacement requirements for the actuator. To assess the feasibility of seat isolation, the inputs to the model must be realistic road disturbances such that the magnitude of the critical parameters (i.e. force and displacement) reflect actual road conditions.

Our paper assesses the feasibility of piezoelectric actuation by first determining an input excitation that reflects realistic road conditions. These inputs are based on the work by Gillespie in the analysis of vehicle dynamics. Road inputs are combined with a statespace representation of the seat-suspension system of a quarter-car model to determine the force and displacement requirements for the piezoelectric actuator. An actuator that meets these specifications is designed and tested on a dead-mass setup that represents the vertical transmissibility of an existing automotive seat.

The first step in the actuator feasibility study is the determination of actuator requirements. The seat and passenger are modeled in a vertical motion, four degree of freedom system which incorporates the seat suspension, effects of the vehicle suspension, as well as a representative input derived from measured road input data. The model output can then be compared to passenger comfort data which has been experimentally determined by various researchers.

\section{Input excitation of the road disturbance}

The input excitations to the seat and vehicle model are modeled with power spectral densities (PSD) of the road disturbances. The energy content in road disturbances has been summarized by Gillespie and is shown in Fig. 1. The PSD amplitude falls with increasing frequency, which is typical of all roads due to the fact that low wavenumber (high wavelength) surface disturbances have larger height variations than do high wavenumber (low wavelength) disturbances. Since acceleration is more commonly used in the measure of ride quality, the road surface elevation variation can be expressed as an acceleration by differentiating twice. The differentiation involves multiplying by frequency, and so the acceleration spectrum now shows a characteristic increase in amplitude with increasing frequency.

Taking a quarter-car model, the road roughness is transmitted through the suspension as illustrated in Fig. 1 [8]. The middle graph is the frequency response function of a typical automotive suspension. Taking the road acceleration input and multiplying it through the suspension response, we finally arrive at the acceleration spectrum at the vehicle level. At the level of the vehicle floorboard, only lower frequency disturbances will be experienced. The vibration that has managed to survive the primary suspension's filtering efforts is now left as the input to the base of the automotive seat. The seat is the final level of isolation between road roughness and the passenger.

\subsection{Passenger comfort}

The performance of the seat is quantified by relating the vibration levels to commonly-accepted indicators of passenger comfort. While there may be no absolute standard of human comfort due to the fact that no two human beings are exactly alike, research has proven that we can define a zone or area in which people generally experience discomfort. Above this region the vibration is certain to be intolerable, and below this region the vibration level is acceptable. In order to quantify this zone, we can begin by simplifying our considerations to a human being subjected to simple sinusoidal motion in one direction. An extensive research summary is given in Gillespie's text, and is reproduced in Fig. 2.

Each line in the figure represents a line of constant comfort above which a test subject will complain of excessive vibration. While the absolute level of vibration at which a person will complain varies from study 

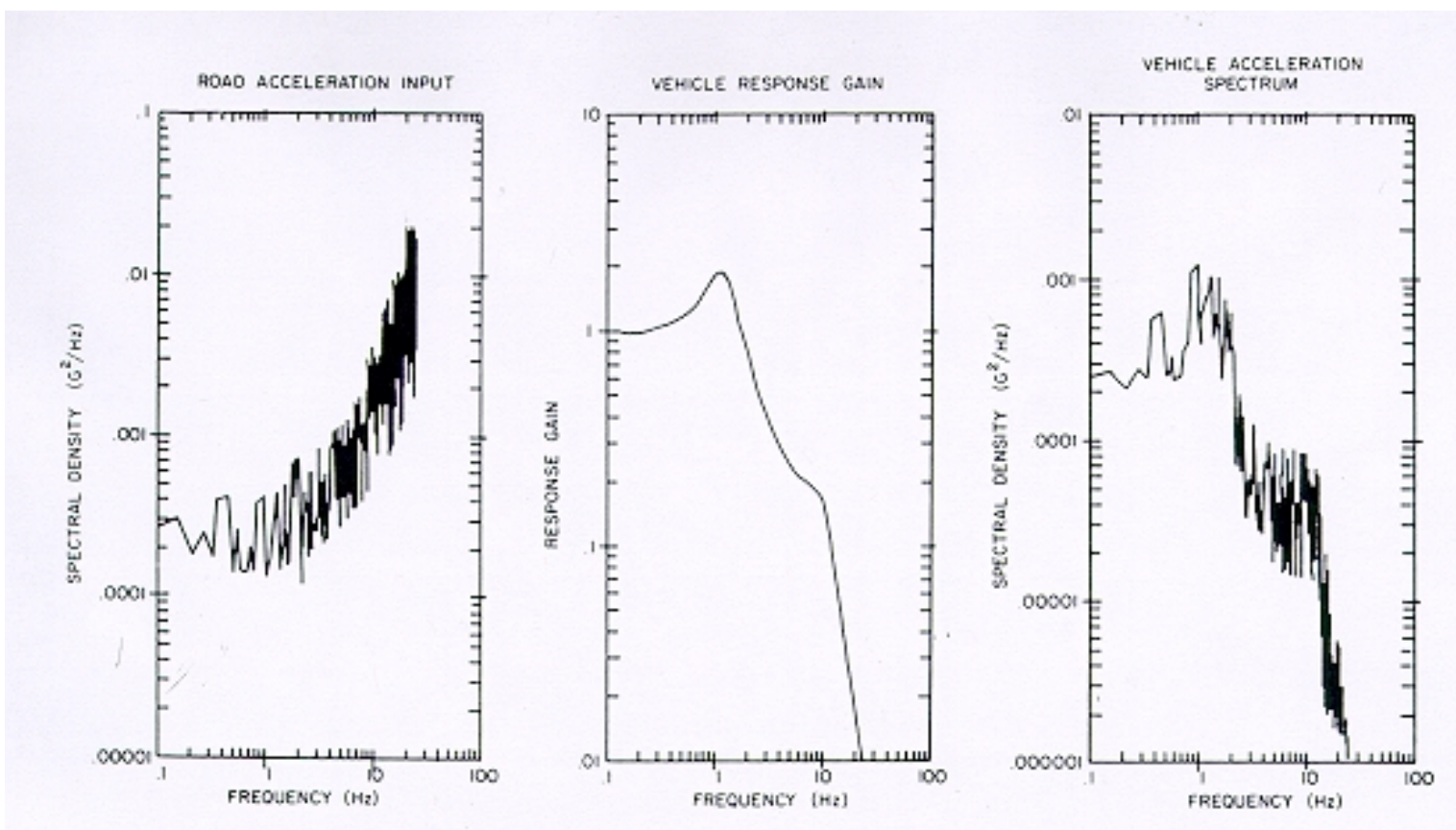

Fig. 1. Power spectral density after seat suspension [8].

to study, the characteristic shape is seen to be similar. Human beings are especially sensitive to vibration in the range of $4-10 \mathrm{~Hz}$, where each curve dips to its minimum. This is often attributed to resonances within the abdominal cavity. Above and below this $4-14 \mathrm{~Hz}$ range, sensitivity decreases and humans are tolerant of higher acceleration levels. The variance seen between researchers is due to differences in several factors, including seating position, compliance of the seat surface used, stated definition of discomfort, subject size, and so on.

Research done by M.J. Griffin exhaustively analyzes comfort contour curves in all three translational directions, as well as rotational excitations about all three axes. His research shows that vibration in the vertical direction causes discomfort at lower vibrational levels for a greater frequency range than do other directional inputs. Griffin's experiments also involve vibrational inputs through the backrest and footrest, with vertical motion of the seat still seen as causing the most discomfort $[9,10]$.

In summary, humans are most tolerant of high frequency vibration, yet the magnitude of road inputs falls off with increasing frequency. Also, vehicle suspensions adequately filter out high frequency inputs, while allowing lower frequencies to pass through to the body of the vehicle. At the interface of the vehicle and the seated human at the seat surface, high frequency vi- bration is of lesser concern than low frequency inputs. These factors are used to assess the performance of the active isolation system.

\section{Seat-car model}

The road excitation PSDs are combined with a lumped parameter model of the seat and car for the purpose of determining force and displacement requirements for the actuator. The model is developed by coupling a quarter-car model with a two-mass-springdamper model of the seat. The resulting four-degreeof-freedom model is shown in Fig. 3. Two degrees of freedom exist due to the unsprung (wheel and tire) mass and the sprung (vehicle frame, body, etc.) mass, and two additional degrees of freedom are added in order to consider the presence of both foam cushion and an active suspension between the passenger and the vehicle. The foam is modeled with a spring rate $K_{f}$ and damping rate $C_{f}$, while the actuator is represented by a spring rate $K_{a}$ in parallel with the force generating actuator. The terms $K_{s}$ and $C_{s}$ represent the springrate and damping of the primary vehicle suspension, and $K_{t}$ represents the tire springrate.

The model allows a great deal of flexibility in the analysis, as various vehicle applications can be considered by modifying the primary suspension spring rate 


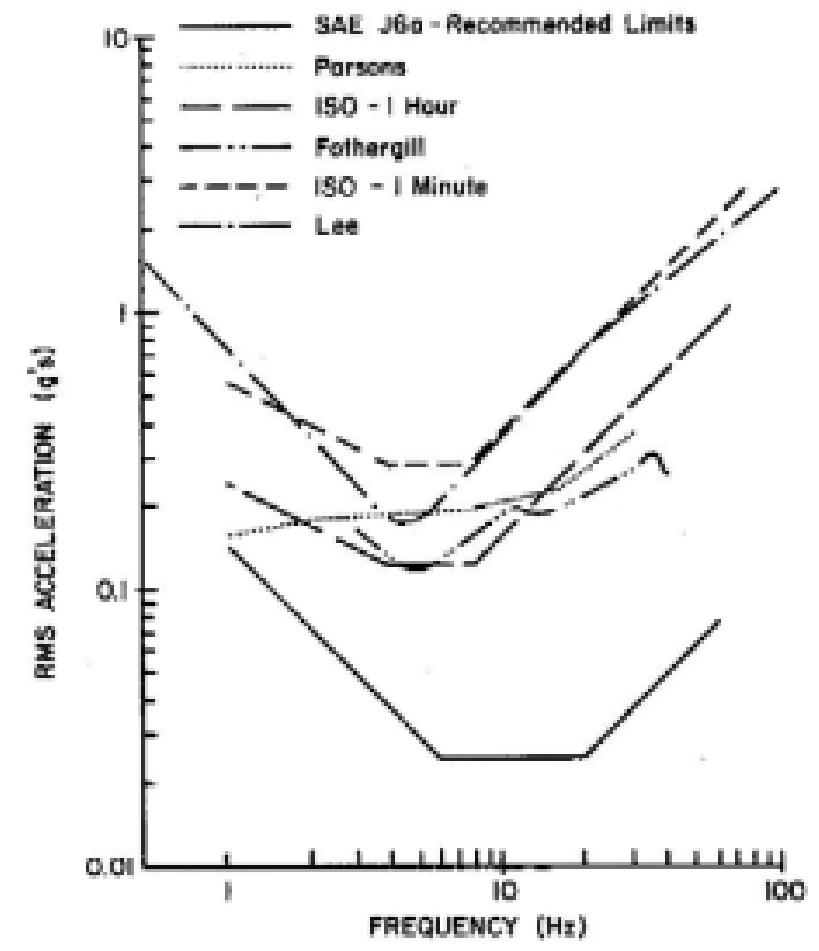

Fig. 2. Tolerance to vertical vibration [7].

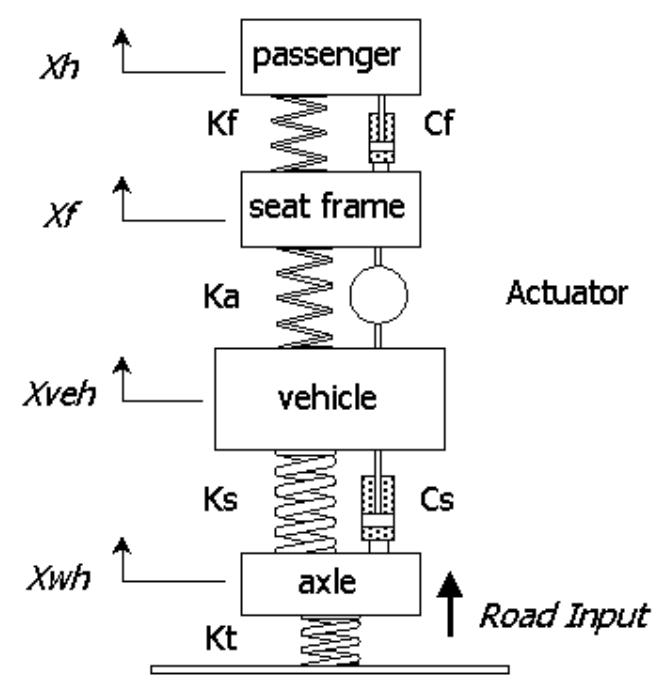

Fig. 3. Four-degree-of-freedom quarter-car and seat model.

and damping ratio. Seat isolation can be evaluated for the case of a foam seat only and a rigid connection in place of the actuator (with $K_{a}$ set very large to imitate infinite stiffness) or a fully active seat with no foam $\left(K_{f}\right.$ set to a very large value). Even when removing foam to save weight in the vehicle, some foam will be required beneath the seated passenger in order to comfortably conform to the contours of the passenger's body. Thus, the lightweight seat application under consideration in this thesis maintains some amount of foam even in the active case.

The pitch and roll modes of the vehicle are not included in the model. Not only is the vertical input the dominant excitation arising from a paved road (in most cases), but in addition, human discomfort is known to be most sensitive to vibration in the vertical direction. Roll excitation has a power spectral density that falls off with decreasing wavenumber, and thus is largely filtered out by the suspension [7]. Pitch may become critically important if the road contains a specific forcing frequency, such as would be present in a fixed slab length concrete road, which excites a pitch resonance of the vehicle. Such a special case is not considered here.

The equations of motion for a position vector $x_{1}$ are given by

$$
x_{1}=\left[\begin{array}{c}
x_{h} \\
x_{f} \\
x_{v e h} \\
x_{w h}
\end{array}\right]
$$




$$
\begin{aligned}
& \begin{array}{c}
\mathrm{F}=\left[\begin{array}{ccc}
0 & 0 & 0 \\
0 & 0 & 0 \\
0 & 0 & 0 \\
0 & 0 & 0 \\
\frac{-k_{f}}{M_{h}} & \frac{k_{f}}{M_{h}} & 0 \\
\frac{k_{f}}{M_{f}} & \frac{-\left(k_{f}+k_{a}\right)}{M_{f}} & \frac{k_{a}}{M_{f}} \\
0 & \frac{k_{a}}{M_{v e h}} & \frac{-\left(k_{s}+\mu_{a}\right)}{M_{v e h}} \\
0 & 0 & \frac{k_{s}}{M_{w h}}
\end{array}\right. \\
{\left[\begin{array}{cccc}
M_{h} & 0 & 0 & 0 \\
0 & M_{f} & 0 & 0 \\
0 & 0 & M_{v e h} & 0 \\
0 & 0 & 0 & M_{w h}
\end{array}\right] \ddot{x_{1}}}
\end{array} \\
& +\left[\begin{array}{cccc}
c_{f} & -c_{f} & 0 & 0 \\
-c_{f} & c_{f} & 0 & 0 \\
0 & 0 & c_{s} & -c_{s} \\
0 & 0 & -c_{s} & \left(c_{s}+c_{t}\right)
\end{array}\right] \dot{x_{1}} \\
& +\left[\begin{array}{cccc}
k_{f} & -k_{f} & 0 & 0 \\
-k_{f}\left(k_{f}+k_{a}\right) & -k_{a} & 0 \\
0 & -k_{a} & \left(k_{s}+k_{a}\right) & -k_{s} \\
0 & 0 & -k_{s} & \left(k_{s}+k_{t}\right)
\end{array}\right] x_{1} \\
& =\left[\begin{array}{l}
0 \\
-F_{a} \\
F_{a} \\
F_{\text {road }}
\end{array}\right]
\end{aligned}
$$

where vertical positions are given by $x_{h}$ for the human passenger, $x_{f}$ for the seat frame, $x_{v e h}$ for the vehicle (sprung mass), and $x_{w h}$ for the wheels and axle (unsprung mass). The equations can then be written in the state space form according to

$$
\begin{aligned}
& \dot{x}=F x+G_{d} u_{d}+G_{c} u_{c} \\
& y_{o}=H_{o} x+J_{o} u_{c} \\
& y_{s}=H_{s} x
\end{aligned}
$$

with variables defined as

$$
\begin{array}{ll}
x & \text { state vector } \\
y_{o}, y_{s} & \text { observation or sensor output vector } \\
u_{d}, u_{c} & \text { disturbance or control input } \\
F & \text { system matrix } \\
G_{d}, G_{c} & \text { disturbance or control input matrix } \\
H_{o}, H_{s} & \text { observation or sensor output matrix } \\
J_{o} & \text { feedforward observation matrix }
\end{array}
$$

The input vector $u$ contains both a defined road input signal, and an actuator input force as determined by the feedback system. The state vector $x$ is now defined as
The state space matrices for the system, according to Eq. (3), become,

$$
\begin{aligned}
& G_{d}=\left[\begin{array}{c}
0 \\
0 \\
0 \\
0 \\
0 \\
0 \\
0 \\
\frac{k_{t}}{M_{w h}}
\end{array}\right] G_{c}=\left[\begin{array}{c}
0 \\
0 \\
0 \\
0 \\
0 \\
\frac{-1}{M_{f}} \\
\frac{1}{M_{v e h}} \\
0
\end{array}\right] \\
& H_{o}=\left[\begin{array}{llllllll}
1 & 0 & 0 & 0 & 0 & 0 & 0 & 0 \\
0 & 0 & 1 & 0 & 0 & 0 & 0 & 0 \\
0 & 0 & 0 & 0 & 0 & 0 & 0 & 0
\end{array}\right] \\
& H_{s}=\left[\begin{array}{cccccccc}
1 & 0 & -1 & 0 & 0 & 0 & 0 & 0 \\
0 & 0 & 0 & 0 & 1 & 0 & -1 & 0
\end{array}\right] \\
& J_{o}=\left[\begin{array}{l}
0 \\
0 \\
1
\end{array}\right]
\end{aligned}
$$

For these particular $H$ and $J$ matrices, the values in the output vector $y$ will be:

$$
\left[\begin{array}{l}
y_{o} \\
y_{s}
\end{array}\right]=\left[\begin{array}{l}
\text { passenger displacement } \\
\text { vehicle displacement } \\
\text { actuator force } \\
\text { passenger displacement } \\
\text { relative to vehicle } \\
\text { passenger velocity relative } \\
\text { to vehicle }
\end{array}\right]
$$

The $J_{o}$ matrix has been chosen to output the actuator force in the third row of $y_{o}$. The $H_{s}$ matrix as shown 


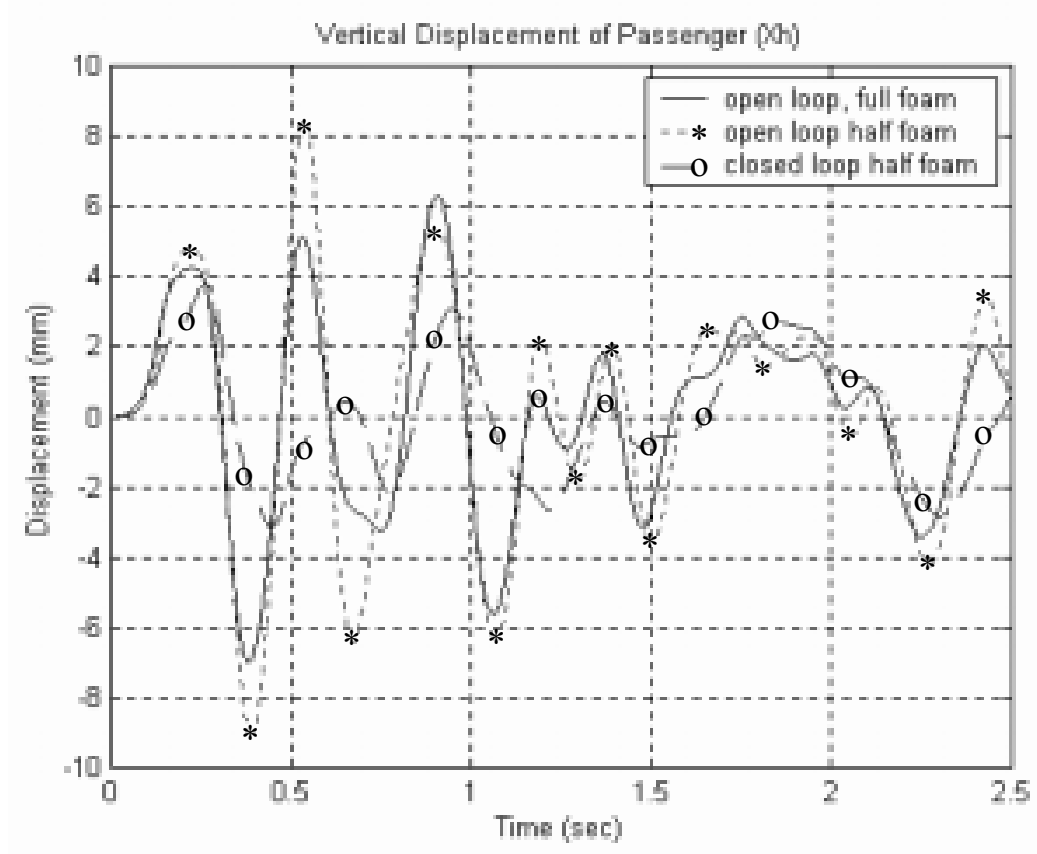

Fig. 4. Simulated time history for passive and active isolation.

here outputs the position difference and velocity difference (of the attachment points of the actuator) in the first and second rows of $y_{s}$, respectively.

With a complete model of the system in the free response condition, the next step is to close the loop and apply a feedback which alters the system response as desired. The feedback system can also be modeled in state space form, with the system matrix now reflecting the actuator dynamics. The expression for feedback control is

$$
u_{c}=-J_{f b} y_{s}
$$

To simply apply feedback gains based upon the output of the model, gains are placed in the $J_{f b}$ matrix. This provides a suitable estimate of required actuator performance.

\section{Simulation results}

Numerical analysis is performed with the parameters shown in Table 1. Known mass values are used for the seat, and the remaining mass values are approximated for a typical small to mid-size sedan type automobile. Spring and damper rates are chosen so as to have a typical primary suspension resonance at $1.5 \mathrm{~Hz}$ and a wheel hop mode at $12 \mathrm{~Hz}$. Seat cushion damping is based upon experimental measurement of an actual passenger seat [13].

Three cases were examined: 1) the open loop, free response of the baseline seat having full foam thickness, 2) the open loop free response of a seat with half the foam removed, and 3) the closed loop response of a seat having half the foam removed which has an active seat suspension added in. Case 2 demonstrates the degradation in vibration isolation that results from removing half of the foam out of the seat, and case 3 represents the attempt at forcing case 2 back to at least the level seen in case 1 . The goal of the active system is to recover the performance lost in removing foam and so case 1 is taken to be the desired level of isolation.

Feedback gains are applied to two outputs of the system - the position difference between the passenger and the vehicle, and the velocity difference between the passenger and the vehicle.

A sample time history of the simulated output is given in Fig. 4, which shows the vertical displacement of the passenger $\left(x_{h}\right)$ for each of the three cases. It is obvious from the figure that removing foam from the seat causes larger excursions in $x_{h}$, which will be experienced by the passenger as a less comfortable ride. The closed loop case demonstrates a marked improvement, and more than recovers the performance lost with the removal of foam from the seat. 

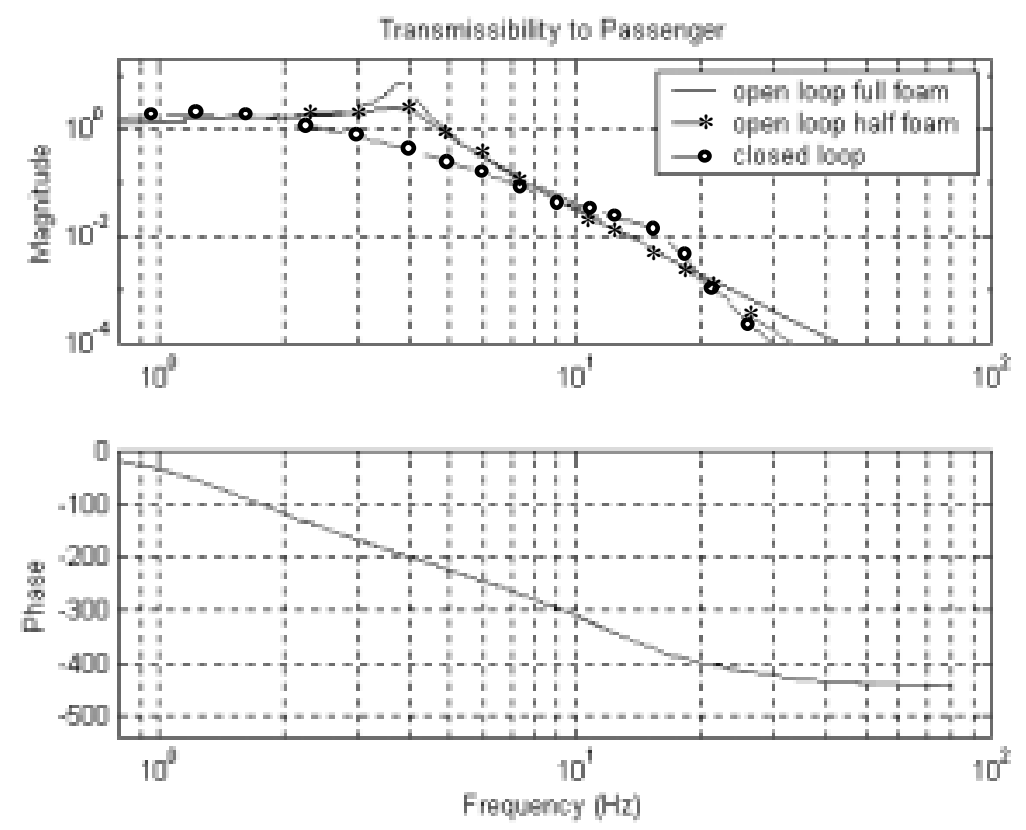

Fig. 5. Simulated frequency response demonstrating the effects of active control on the seat transmissibility.

Figure 5 shows the frequency response function for all three cases. With the decrease in foam thickness, the performance loss is seen as a higher peak at both system resonances. This is due to the loss of damping with the decrease in foam thickness. Note that the resonant frequencies remain constant, since in the half foam case the spring in parallel with the actuator is assumed to recover the loss in spring rate with the removal of foam. The spring shown by $K_{a}$ in Fig. 3 is necessary as it offloads the actuator by supporting the static weight of the seat and passenger. The actuator then needs only to apply force for the dynamic movement of the mass, and not to support the mass in a static condition. This greatly decreases actuator force requirements. With the actuator incorporated in feedback control of the system, a performance improvement is obvious in Fig. 5. The isolation frequency of the system has been decreased from $5 \mathrm{~Hz}$ to $2.5 \mathrm{~Hz}$, and the transmissibility at both resonant frequencies has been decreased.

Now that the $J_{f b}$ matrix has been determined for a sufficient level of performance, actuator force and stroke can be extracted from the simulation results. Figure 6 shows a time history of both actuator force, in Newtons, and actuator stroke, in millimeters. Actuator force can be seen to reach peaks of approximately \pm 400 Newtons, with actuator stroke reaching \pm 4 millimeters. RMS actuator force is $288 \mathrm{~N}$, and RMS actuator stroke is $2.9 \mathrm{~mm}$. Results are summarized in Table 2.

\section{Actuator design and testing}

The actuator is designed using piezoelectric materials because of their ability to achieve the force levels required of the isolation application. Piezoelectric actuators have only $0.1 \%$ maximum strain, therefore one of the primary design considerations is determining an actuator configuration that achieves the millimeterrange displacements estimated by the numerical simulations. As discussed in Near [18], there are a number of methods of increasing the stroke of piezoelectric actuators. For this work we have chosen to use a prestressed, curved piezoelectric actuator sold by Face International Corporation. The rationale for this choice is twofold:

1. A recent study by Berner and Brei [4] demonstrate that this type of actuator has a high energy output compared to other types of large-stroke configurations.

2. Their force and displacement ratings are in the range for this application and they are commercially available.

The key elements of the design and testing of the actuator are discussed in the following two sections.

\subsection{Piezoceramic actuator implementation}

While the prestressed piezoceramic devices exhibit force and displacement levels near the requirements of 

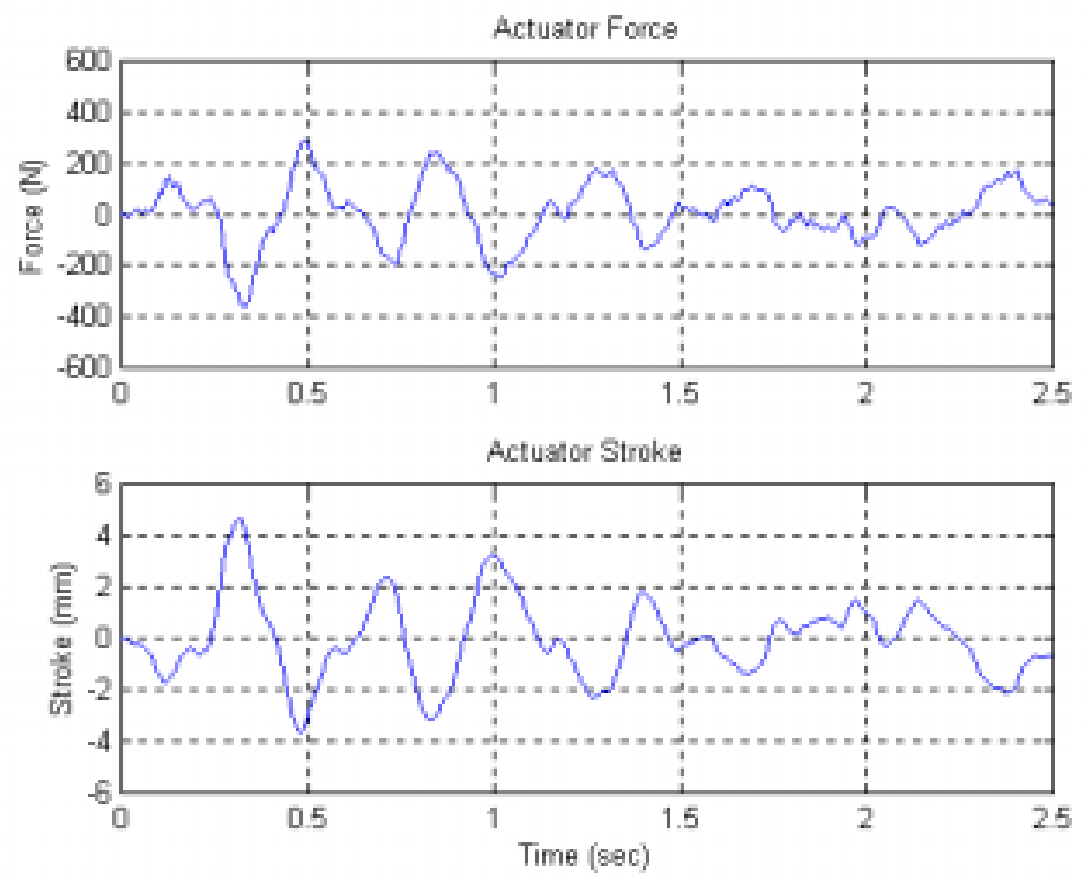

Fig. 6. Force and stroke levels computed from the car and seat simulations.

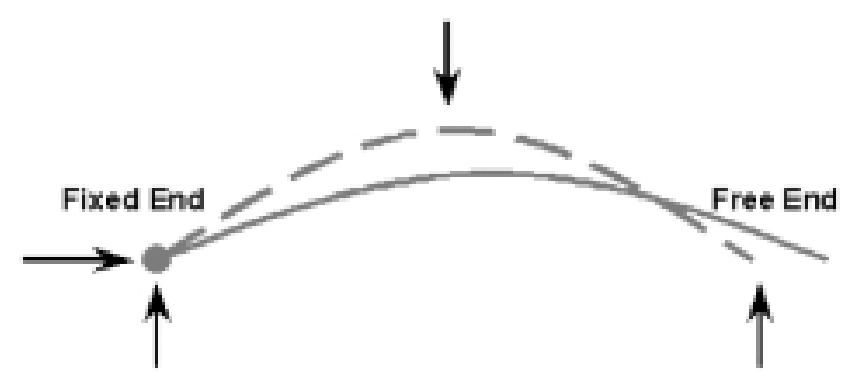

Fig. 7. Actuator endpoint movement under deflection.

the isolation application, the actual implementation into a mechanical system is far from simple. Vertical displacement at the midpoint of the arch is developed by a change in radius of curvature, which also carries with it a corresponding horizontal movement of the endpoints. There are three distinct load - bearing points - one at the center and one at each end. In the horizontal direction, one of these must be constrained to keep the system in place, but the other two must be allowed to slide freely in order to accommodate the length change that accompanies change in curvature. All three attachment points must be constrained in the vertical direction, since this is the direction in which force is to be applied to the seat system. Figure 7 demonstrates the situation, with the arrows representing the free body reaction forces required in the mounting scheme. In addition, the fixed end must allow rotational movement due to the small angular change that accompanies deflection.

In this application, two stacked actuators will be used in order to increase force level. A thin Teflon sheet placed between the two devices serves to electrically isolate the two oppositely charged surfaces, and also to permit the small amount of sliding that will occur. The midpoint of the actuator will be attached to the seat frame, while the endpoints will be attached to the floorboard, with a static weight supporting spring in parallel with the actuator assembly. Figure 8 shows the incorporation of all necessary features into the actuator components and the assembled actuator. In the as- 

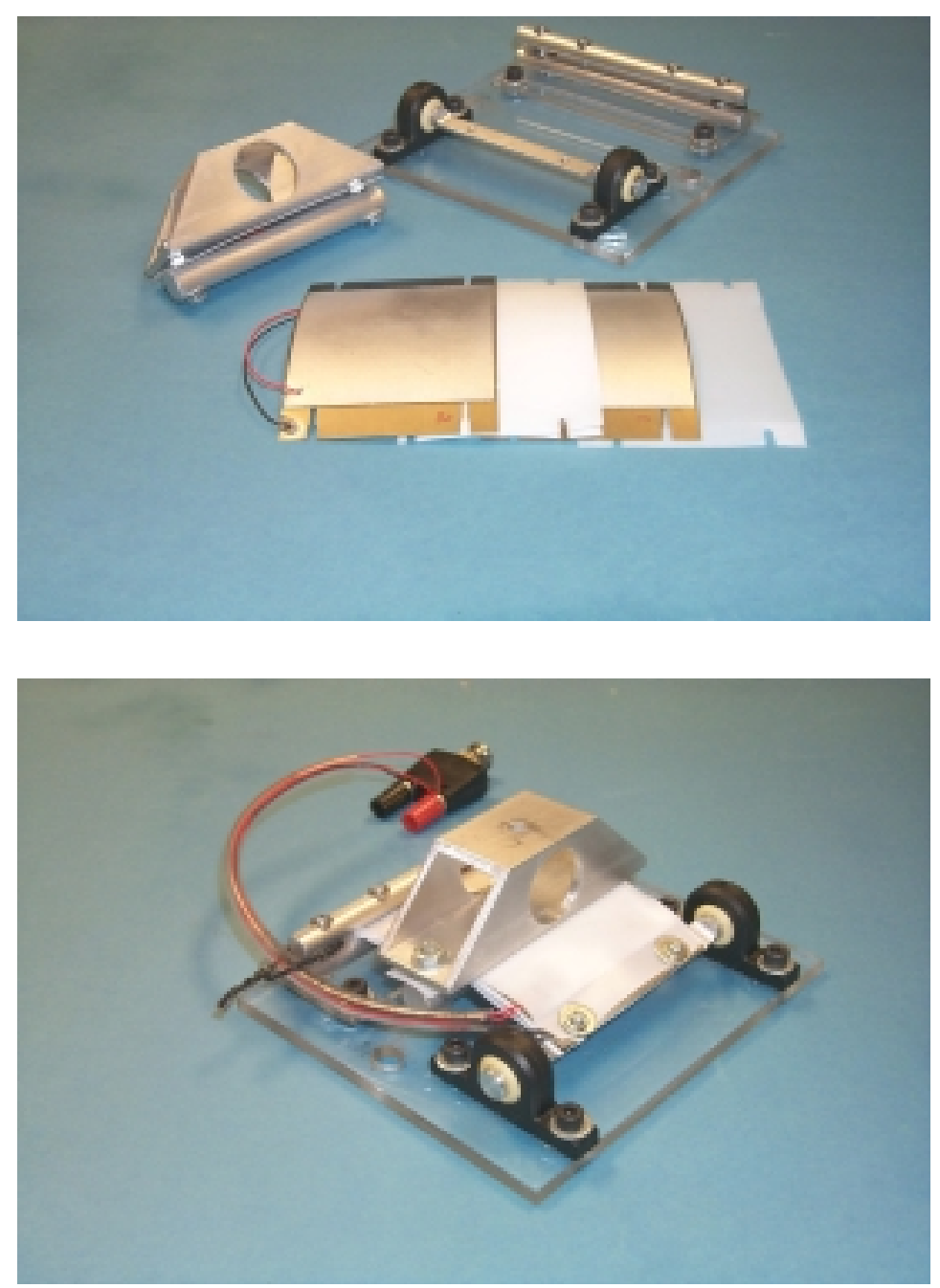

Fig. 8. Actuator assembly.

tested configuration, the complete assembly weighs a total of 488 grams.

\section{Free displacement and blocked force measurements}

Free displacement is measured by applying a voltage signal to the actuator, in the absence of any external load, and measuring displacement. A sinusoidal input voltage is applied at frequencies of $1 \mathrm{~Hz}, 5 \mathrm{~Hz}$, and $10 \mathrm{~Hz}$. Since input voltage is limited to $+600 \mathrm{~V}$ and $-300 \mathrm{~V}, \mathrm{a}+150 \mathrm{~V}$ offset is applied in order to maximize peak to peak voltage range. A Polytech OFV 303 laser vibrometer is used to measure displacement. Test setup and the measured results are shown in Fig. 9. Maximum stroke is $4.4 \mathrm{~mm}$ peak to peak for an input of $900 \mathrm{~V}$ peak to peak. The data shows a reasonably linear response beyond $300 \mathrm{~V}$, with a gain of $0.00625 \mathrm{~mm} / \mathrm{V}$.
At the other extreme of the operating envelope is the case of zero displacement with maximum actuator force, or blocked force. The actuator is constrained to prevent any displacement, energized, and blocked force is measured (see Fig. 10) with a quartz force transducer is affixed between the actuator and the plate. Peak to peak input voltage is varied from 0 to $900 \mathrm{~V}$, in increments of $50 \mathrm{~V}$, with data taken for sinusoids of $1 \mathrm{~Hz}, 5 \mathrm{~Hz}$, and $10 \mathrm{~Hz}$. Figure 10 shows a maximum of $320 \mathrm{~N}$ peak to peak. Again, response is linear beyond the $300 \mathrm{~V}$ level, with a gain of $0.41 \mathrm{~N} / \mathrm{V}$.

\section{Control experiment}

The simplified case of a single actuator assembly controlling the vertical motion of a representative 

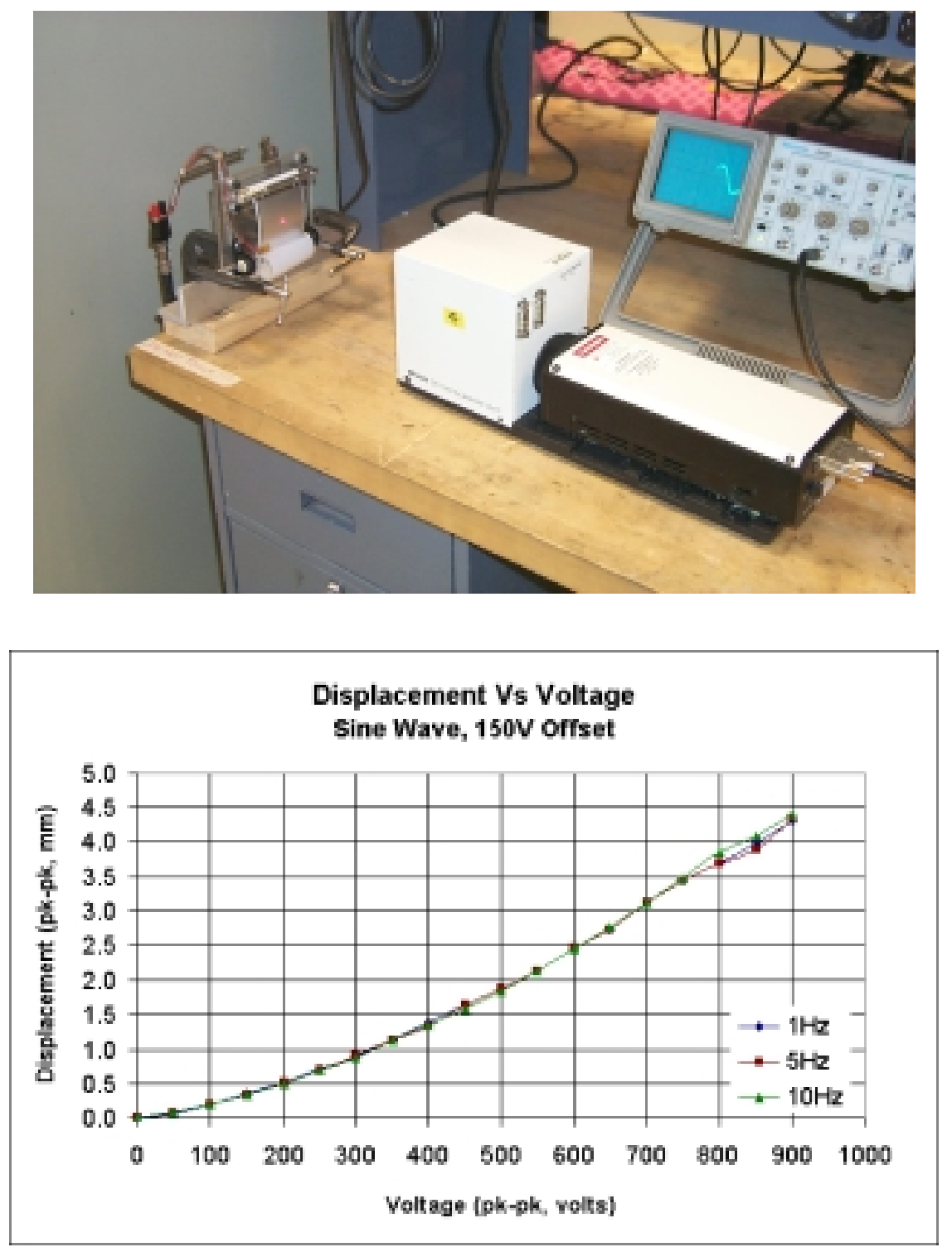

Fig. 9. Experimental setup from free displacement measurement (top) and measured actuator free displacement versus voltage (bottom).

amount of dead mass can now be examined. A vertical motion test stand driven by an electrodynamic shaker is employed to analyze the system response. Since only one actuator assembly is being tested, one quarter of the total mass is used. This amounts to $24.4 \mathrm{~kg}$, which is one fourth of the $21 \mathrm{~kg}$ seat mass plus $77 \mathrm{~kg}$ of human mass.

The setup is shown is Fig. 11. The steel mass is constrained to vertical motion by means of the linear bearings sliding along the aluminum posts on either side, with the actuator assembly directly below the dead mass, situated on an adjustable height platform. The two static weight supporting springs in parallel with the actuator are readily visible in the picture, as are the two accelerometers (one on top of the mass, one on the table surface directly below the actuator) used to measure transmissibility. The calculated natural frequency of the $24.4 \mathrm{~kg}$ dead mass with two $12.3 \mathrm{~N} / \mathrm{mm}$ static weight springs is $5.05 \mathrm{~Hz}$. This does not account for the added spring rate of the piezoceramic devices themselves, which will raise the resonant frequency slightly. The stiffness of the springs in the test setup have been chosen so that the natural frequency of the mass-spring system is approximately equal to the resonance deter- 

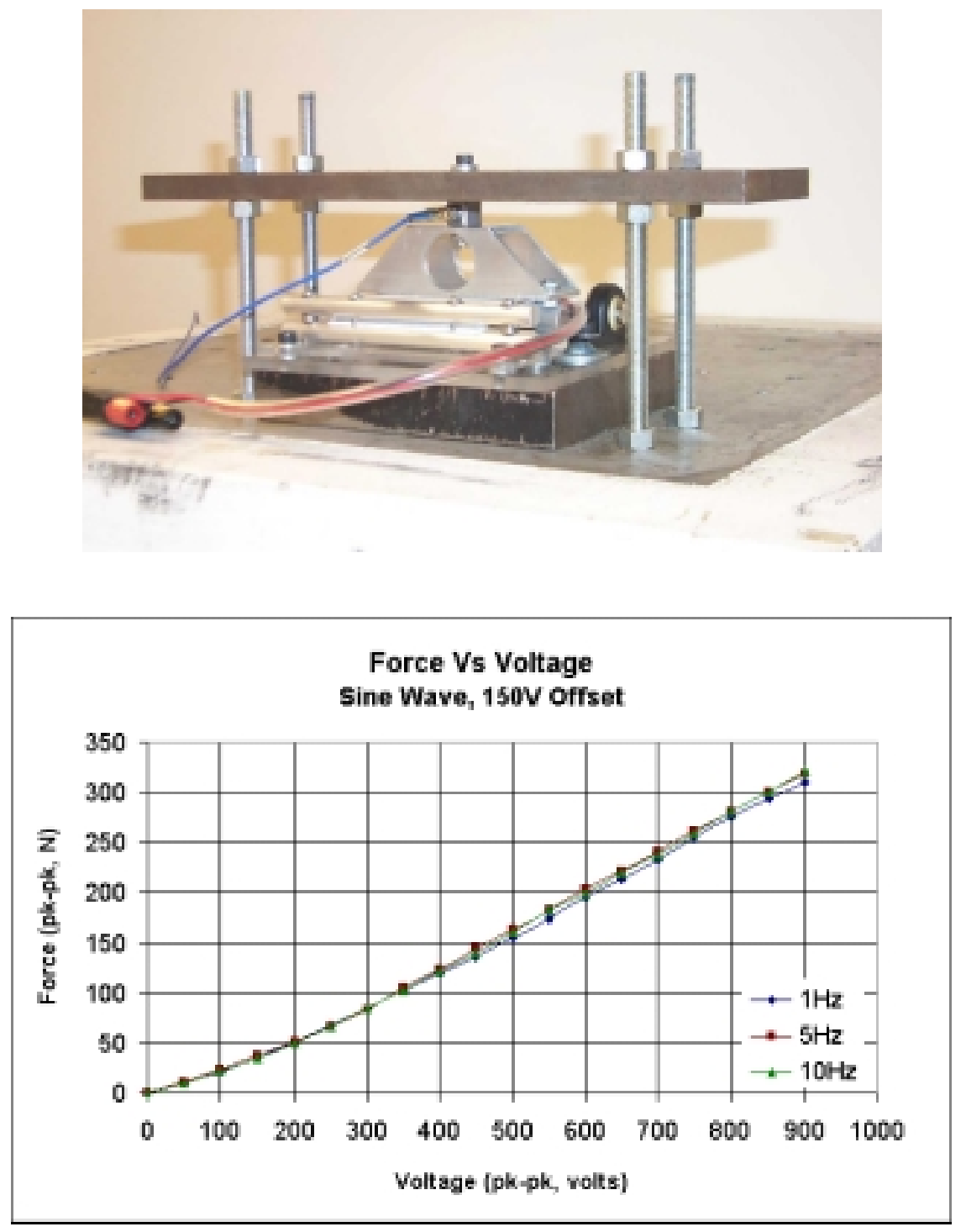

Fig. 10. Experimental setup for blocked force measurement (top) and measured blocked force versus voltage.

mined from transmissibility tests on the actual seat. In an actual design, the springs also serve to offload the static weight of the passenger and the seat for the purpose of reducing the force requirements of the piezoelectric actuator. Over a range of weights (e.g. for different passengers), the force capability of the actuator would be reduced due to the additional static load placed on the actuator. Although this is a practical design consideration, this aspect of the problem was not studied in our work.

To produce a shaker table noise signal that mimics the floor of a vehicle driving down the road, the dSpace digital signal processing system is used for signal generation and data acquisition. The representative road input is simulated using a bandwidth - limited noise signal filtered through two digital transfer functions. The first transfer function converts the noise from a broad spectrum noise to a signal that is representative of an actual road surface. A second transfer function, representing the vehicle primary suspension, is used to convert the road noise to a vehicle floorboard noise. The final signal is fed into the dSpace digital to analog converter, and the actual output voltage signal is fed into the amplifier driving the electrodynamic shaker. The resulting input signal contains only lower frequency content, just as a typical road would. This signal is used to determine the effect of feedback control the seat vibration resulting from a representative road profile. 


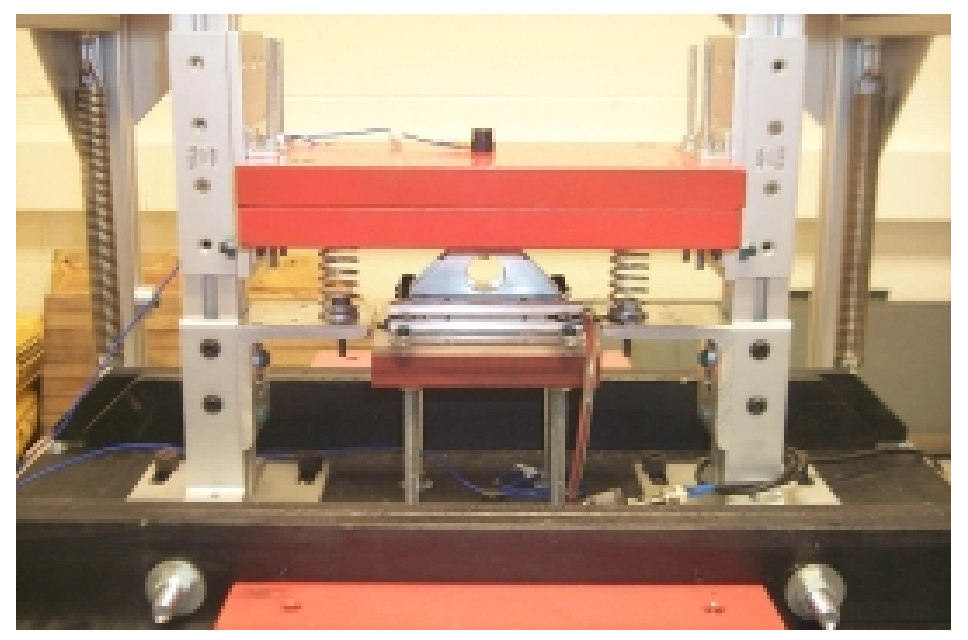

Fig. 11. Dead mass setup on test stand.
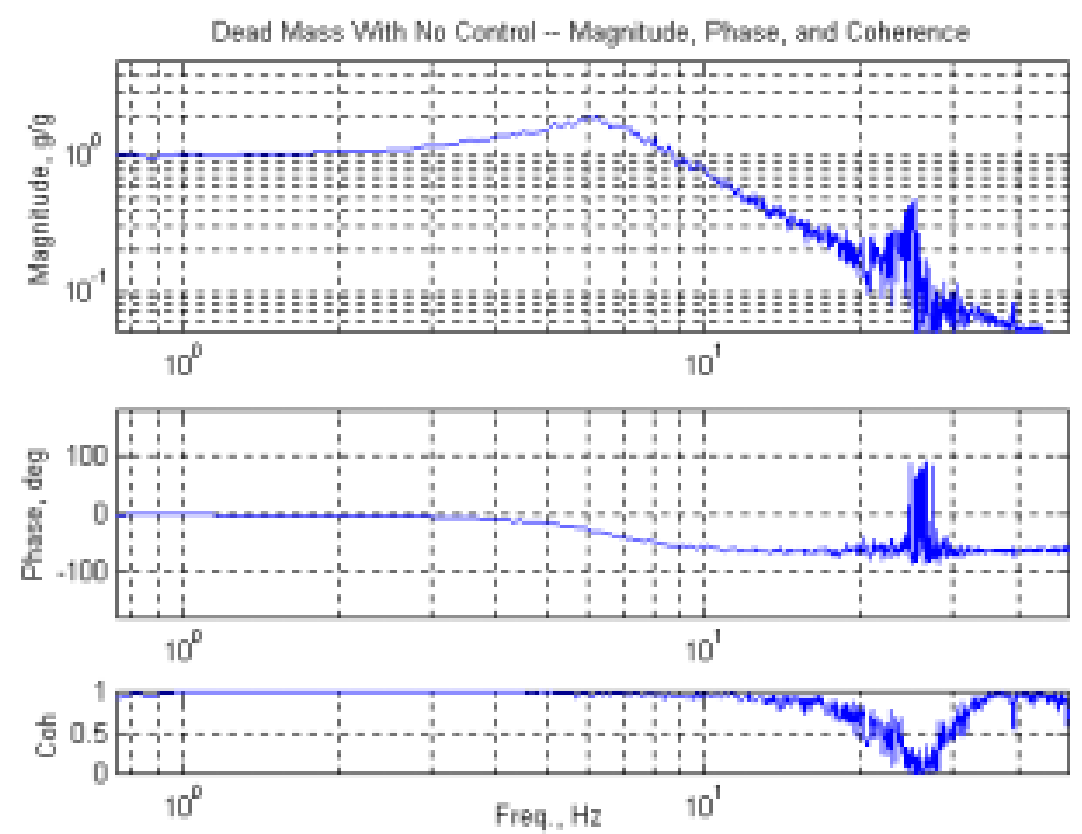

Fig. 12. Frequency response between the floor vibration and the mass motion: magnitude (top), phase (middle), and coherence (bottom).

Upon taking the frequency response function (FRF), the coherence in the higher end of the frequency range is very low. The filtering has done such a good job screening out all of the higher frequency content of the noise signal that there is only negligible acceleration in the frequency range above $10 \mathrm{~Hz}$ to excite the system. When the input signal is so small, any noise in the accelerometers, wires, or instrumentation becomes large in comparison, and results in low coherence. To remedy this, a broadband input signal is used to excite the shaker table during the frequency domain analysis. The broadband excitation allows us to determine the transfer function between the floor vibration and the motion of the mass. The shaker table now has an adequate input signal throughout the analysis frequency range. The dips in the $6 \mathrm{~Hz}$ and $25 \mathrm{~Hz}$ range are attributable to the energy being absorbed by resonances of the table and dead mass system.

With the broadband input signal, a suitable FRF of the system is obtained, as shown in Fig. 12. The res- 

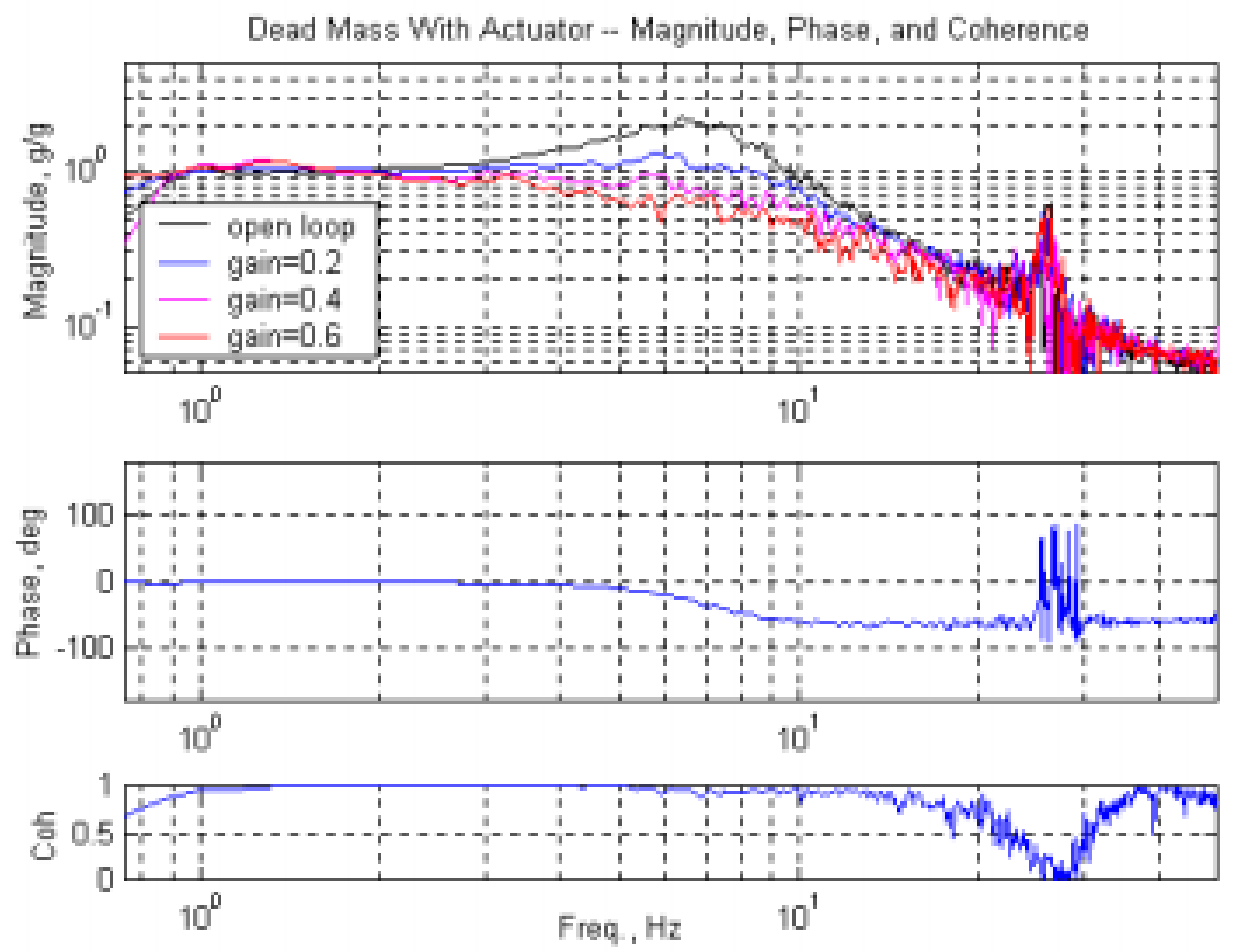

Fig. 13. Measured transmissibility plots illustrating the effects of feedback control.

onance of the system is at $6 \mathrm{~Hz}$, with an isolation frequency of $9 \mathrm{~Hz}$. The poor coherence at $25 \mathrm{~Hz}$ is due to a resonance of the surface of the shaker table itself, which was determined by moving the output accelerometer to various locations, and noting that the table surface itself resonates at $25 \mathrm{~Hz}$.

The dead mass setup allows us to perform active vibration isolation experiments with inputs that are representative of actual road profiles. Active control is implemented through feedback of the mass velocity to the input of the piezoelectric actuator. A velocity signal is obtained by measuring the mass acceleration directly and digitally-integrating the signal in the digital signal processor. The integrated signal is then amplified and output to the input of the actuator.

Figure 13 illustrates the effect of velocity feedback on the transmissibility between the floor and the mass. The resonant peak is eliminated by even modest gains, and as gain is increased further, isolation frequencies in the $2-3 \mathrm{~Hz}$ range are easily achievable. The gain is increased up to a level showing significant clipping at the maximum allowable actuator voltage of $+600 \mathrm{~V}$ and $-300 \mathrm{~V}$.

The experiments also demonstrate that low-frequency vibration isolation is achieved without an increase in the transmissibility at high frequencies. The frequency at which the transmissibility falls below 1 is reduced from approximately $9.5 \mathrm{~Hz}$ in the uncontrolled system to $2 \mathrm{~Hz}$ with maximum feedback gain. The decrease in the isolation frequency is not accompanied by an increase in the transmissibility at high frequencies, as would be the case for increasing the damping coefficient of a purely passive isolation system.

The reduction in RMS acceleration produced by the control used in Fig. 13 is given in Table 3. The table also shows the reduction in RMS acceleration achieved by the numerical simulation for the modeled foam seat verses the modeled active seat, which produced an $80 \%$ reduction in acceleration level. The dead mass experimental setup did not include a test of actual seat foam, and so a direct comparison cannot be made for the passive cases, but the active cases can be compared directly. The input acceleration level was normalized such that both the modeled and the experimental cases experienced the same level of acceleration input. A more than adequate level of isolation is achieved.

A typical automotive seat has a resonant peak at $4 \mathrm{~Hz}$ with an isolation frequency at $6 \mathrm{~Hz}$ [13]. At $4 \mathrm{~Hz}$, the seat has a magnitude ratio of two, meaning any signal near $4 \mathrm{~Hz}$ that manages to pass through the 

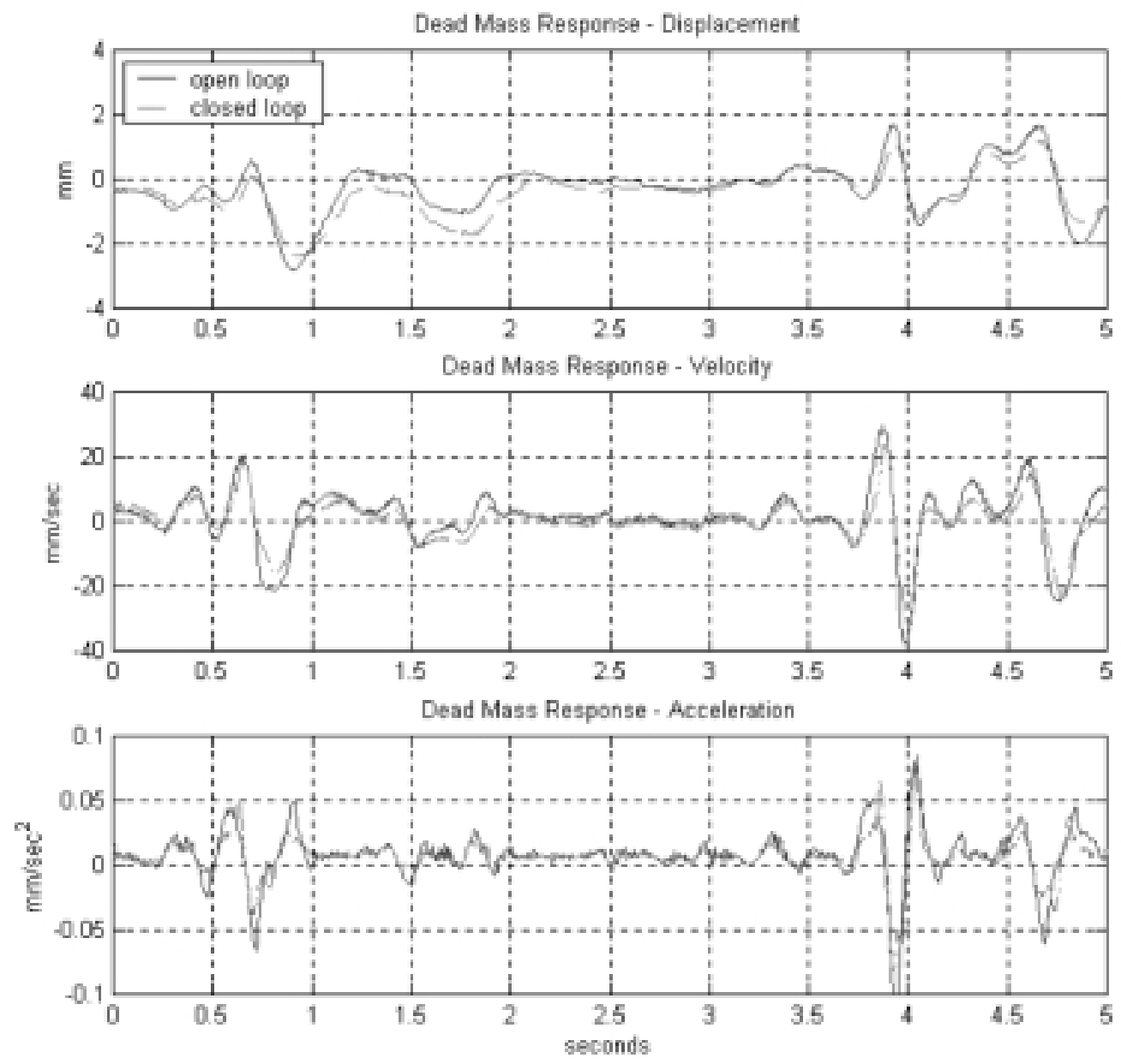

Fig. 14. Measured time history of the dead mass.

primary suspension will actually be amplified by the baseline seat. This is one of the advantages of active suspension systems - Fig. 13 clearly shows that it is possible to actively control the system to eliminate any amplification.

A time history of the dead mass setup being driven with a representative road input signal is shown in Fig. 14. Both the open loop and closed loop responses are shown, with the active case having an absolute velocity gain of 0.5 . Identical random inputs are obtained for the two cases by using the exact same random signal. The results are not as drastic as predicted by the model, since greater effort was made to obtain a representative road signal in the dead mass experiment than was made in the simulation. The active system shows greatest improvement over the baseline seat in the 4$10 \mathrm{~Hz}$ region, while the frequency content of the representative road signal begins to fall off above $3-4 \mathrm{~Hz}$.
The representative road input does not incorporate distinct road events such as potholes or railroad tracks which might better emphasize the improvement of the active system.

\section{Conclusions}

Prestressed, curved piezoceramic actuators are successfully applied to the problem of achieving vibration isolation of an experimental setup representing onequarter of a vehicle seat and passenger. The active system eliminates any amplification in the frequency response function, whereas the baseline seat tested has a magnitude ratio of two at its resonant frequency of $4 \mathrm{~Hz}$. Isolation frequency is reduced to $2-3 \mathrm{~Hz}$, while the baseline seat does not begin isolation until $9.5 \mathrm{~Hz}$. This was accomplished with the design of a piezoce- 
ramic actuator that required millimeter-range displacements and forces on the order of $300 \mathrm{~N}$.

The passenger comfort data suggests that a car seat with a resonant peak in the $4-10 \mathrm{~Hz}$ region is directionally incorrect, since this is the frequency range in which human beings are most sensitive to vertical vibration. By eliminating any amplification in this range, the potential for a passenger to experience discomfort is reduced. This work demonstrates that velocity feedback of the seat motion produces a highly damped system that exhibits no resonant amplification and maintains desirable high-frequency isolation characteristics.

\section{Acknowledgements}

The authors gratefully acknowledge the support of DaimlerChrysler, program coordinator Mr. Steve Buckley.

\section{References}

[1] Y. Ahn, M. Ahmadian and S. Morishita, On the design and development of a magneto-rheological mount, Vehicle System Dynamics 32, (1999), 199-217.

[2] E.H. Anderson, D.J. Leo and M.D. Holcomb, Active system for vibration isolation of spacecraft instruments, in Proceedings of the Rocky Mountain Guidance and Control Conference, 1996, pp. 465-480.

[3] A. Beard, D. Flotow and A. von Flotow, Practical product implementation of active-passive vibration isolation, Proceedings of the SPIE 2264 (1994), 38-49.

[4] N.T. Berner and D.E. Brei, Modeling and study of the quasistatic behavior of piezoceramic telescopy actuation architectures, ASME Adaptive Structures and Material Systems AD-59 (1999), 189-197.
[5] G. Bohannan, H. Schmidt, D. Brandt and M. Booibroek, Piezoelectric polymer actuators for active vibration isolation in space applications, Ferroelectrics 224 (1999), 639-647.

[6] J. Ervin and D.E. Brei, Modeling and experimental characterization of the dynamic behavior of piezoelectric recurve actuator, Proceedings of the ASME Design Engineering Technical Conference 7 (1999), 2391-2402.

[7] T.D. Gillespie, Heavy Truck Ride, Society Of Automotive Engineers, Inc., 1985.

[8] T.D. Gillespie, Fundamentals Of Vehicle Dynamics, Society Of Automotive Engineers, Inc., 1992.

[9] M. Griffin, K. Parsons and E. Whitham, Vibration and control, i translational seat vibration, Ergonomics 25 (1982), 603-630.

[10] M. Griffin, K. Parsons and E. Whitham, Vibration and control, iv application of experimental results, Ergonomics 25 (1982), 721-739.

[11] D.J. Leo and D.J. Inman, A quadratic programming approach to active-passive vibration isolation, Journal of Sound and Vibration 220 (1999), 807-825.

[12] D.J. Leo, M. Malowicki, S.J. Buckley and N. Naganathan, Active seats for hybrid-electric vehicles, Proceedings of SPIE 3674 (1999), 118-126.

[13] M. Malowicki, Active vibration isolation using induced strain actuators with application to automotive seat suspensions, Master's thesis, Virginia Polytechnic Institute and State University, 2000

[14] L. Mauck and C.S. Lynch, Piezoelectric hydraulic pump, Proceedings of the SPIE 3668 (1999), 844-852.

[15] L. Mauck and C.S. Lynch, Piezoelectric hydraulic pump development, Proceedings of the SPIE (2000), 3985-4085.

[16] L.R. Miller, M. Ahmadian, C. Nobles and D. Swanson, Modelling and performance of an experimental active vibration isolator, Journal of Vibration and Acoustics 117 (1995), 272.

[17] K. Nasser, D.J. Leo and H.H. Cudney, Compact piezohydraulic actuation system, Proceedings of the SPIE (2000), 3991-4041.

[18] C.D. Near, Piezoelectric actuator technology, Proceedings of the Smart Structures and Materials Conference 2717 (1996), 246-258.

[19] J.M. Sullivan, Z. Rahman, R. Cobb and J. Spanos, Closed-loop performance of a vibration isolation and suppression system, Proceedings of the American Control Conference 3 (1997), 3974. 

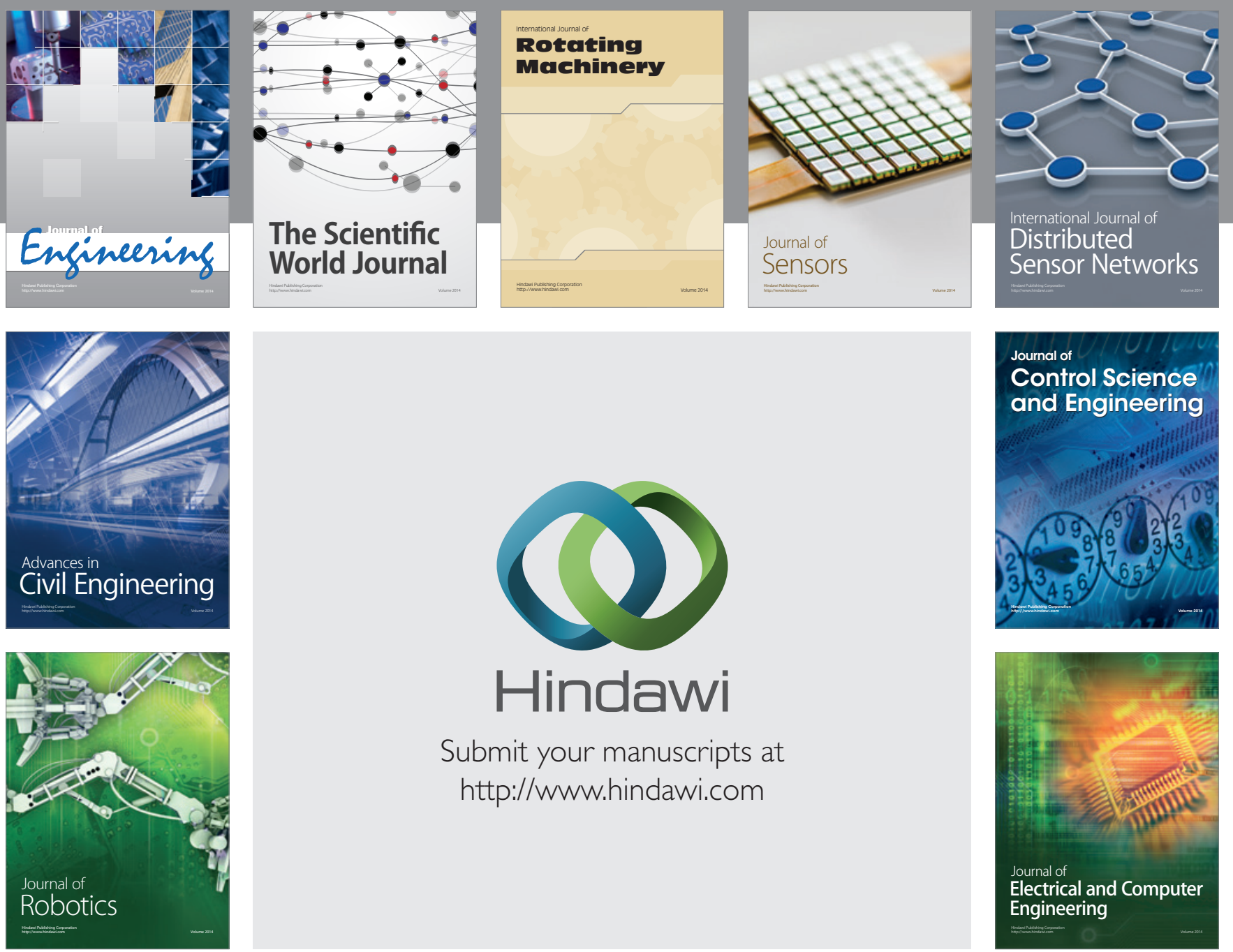

Submit your manuscripts at

http://www.hindawi.com
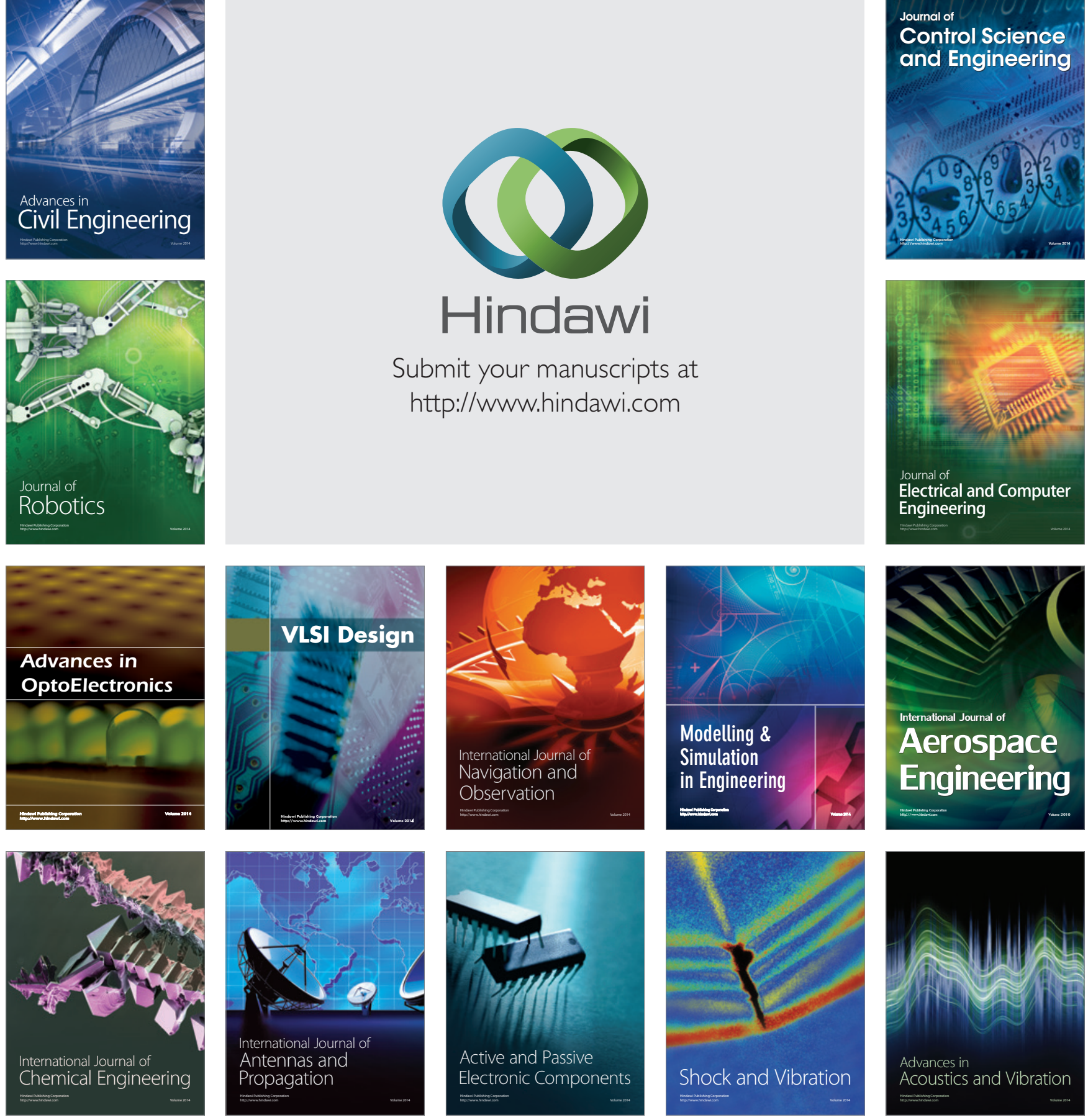